\title{
Fuzzy Sliding Mode Controller Design Using Takagi-Sugeno Modelled Nonlinear Systems
}

\author{
S. Bououden, ${ }^{1}$ M. Chadli, ${ }^{2}$ and H. R. Karimi ${ }^{3}$ \\ ${ }^{1}$ Laboratoire d'Automatique et de Robotique, Université de Constantine, Route d'Ain El Bey, Constantine, Algeria \\ ${ }^{2}$ MIS (EA 4290), University of Picardie Jules Verne, 33 rue Saint-Leu, 80039 Amiens, France \\ ${ }^{3}$ Department of Engineering, Faculty of Engineering and Science, University of Agder, \\ 4898 Grimstad, Norway \\ Correspondence should be addressed to M. Chadli; mohammed.chadli@u-picardie.fr
}

Received 4 September 2012; Accepted 4 October 2012

Academic Editor: Peng Shi

Copyright $\odot 2013$ S. Bououden et al. This is an open access article distributed under the Creative Commons Attribution License, which permits unrestricted use, distribution, and reproduction in any medium, provided the original work is properly cited.

Adaptive fuzzy sliding mode controller for a class of uncertain nonlinear systems is proposed in this paper. The unknown system dynamics and upper bounds of the minimum approximation errors are adaptively updated with stabilizing adaptive laws. The closed-loop system driven by the proposed controllers is shown to be stable with all the adaptation parameters being bounded. The performance and stability of the proposed control system are achieved analytically using the Lyapunov stability theory. Simulations show that the proposed controller performs well and exhibits good performance.

\section{Introduction}

Recent research on fuzzy logic control has, therefore, been devoted to model based fuzzy control systems that guarantee not only stability, but also performance of closed-loop fuzzy control systems [1-6]. For a systematic control design of nonlinear systems, the Takagi-Sugeno (T-S) fuzzy model [4, $5,7-12]$ has been a popular choice in industrial processes due to its ability to represent the nonlinear system only for inputoutput data without complex mathematical equations.

In an effort to improve the robustness of the adaptive fuzzy control system, many works have been published on the design of adaptive fuzzy sliding mode controller [13$18]$, which integrates the sliding mode controller $[16,19-23]$ design technique into the adaptive fuzzy control to improve the stability and the robustness of the control system. Conventionally, adaptive fuzzy sliding control systems (AFSCSs) design is based on the assumption that the system states are available for measurement, so the adaptive laws of AFSCS are formulated as functions of the tracking error of the system [21, 24-27].

However, some problems on the algorithm convergence and conditions stabilities remain with no response. To resolve this problem, first is the need for accurate information on the evolution of the system in the state space, upper bounds of uncertainties and disturbances. The second is the knowledge of the upper bound of the minimum approximation error. We know that the uncertain nature of nonlinear systems makes it difficult to have an analytical description of the dynamics of the system. Moreover, the knowledge of the upper bound of the minimum approximation error leaves the control law still restrictive. In the further study involving a perturbed largescale system with a time-varying interconnection, an adaptive algorithm for estimating an uncertain upper bound based on a variable sliding control frame was proposed in [28].

In this note, based on the variable surface, a fuzzy sliding model controller is developed for guaranteeing the tracking performance, in particular, time-varying uncertain parameters are approximated by fuzzy system, and the adaptive sliding mode control is designed so as to compensate for any unknown reconstruction error, through parameter adaptation law. In this way, the actual system can follow the reference signal even in the event of a hard nonlinearity, and fuzzy sliding mode control gives the unknown upper bound of uncertainties that are adaptively updated with stabilizing adaptive laws. It is proved that the closed-loop system is 
globally stable in the Lyapunov sense if the signals involved are bounded and the system output can track the desired reference input asymptotically.

This paper is organized as follows: some preliminaries are provided in Section 2. Following the introduction, the fuzzy logic system is reviewed briefly in Section 3. The design and stability analysis for the proposed adaptive fuzzy sliding mode controller is included in Section 4. Simulation examples to demonstrate the performance of the proposed method are provided in Section 5. Finally, in Section 6, we give a brief conclusion.

\section{Preliminaries}

Consider the $n$ th-order nonlinear dynamical system of the form:

$$
\begin{gathered}
x^{n}=f(\mathbf{x})+g(\mathbf{x}) u+d_{s}, \\
y=x,
\end{gathered}
$$

where $\mathbf{x}=\left[x, \dot{x}, \ldots, x^{n-1}\right]^{T}=\left[x_{1}, x_{2}, \ldots, x_{n}\right]^{T} \in R^{n}$ is vector of the system that is assumed to be available for measurements, $f$ and $g$ are unknown but bounded nonlinear functions, $u \in R$ and $y \in R$ control input and output of the system, respectively, and $d_{s}$ is external disturbance. As system (1) is required to be controllable, the nonzero condition of input gain $g(\mathbf{x}) \neq 0$ is necessary.

The system (1) can be rewritten in the following form:

$$
0=-g^{-1}(\mathbf{x}) x^{n}+g^{-1}(\mathbf{x}) f(\mathbf{x})+u+g^{-1}(\mathbf{x}) d_{s} .
$$

By adding $x^{n}$ to both sides, we get

$$
x^{n}=x^{n}-g^{-1}(\mathbf{x}) x^{n}+g^{-1}(\mathbf{x}) f(\mathbf{x})+u+g^{-1}(\mathbf{x}) d_{s} .
$$

Equation (3) can be rewritten as

$$
x^{n}=F(\mathbf{x})+u+d(\mathbf{x})
$$

such that

$$
\begin{gathered}
F(\mathbf{x})=\left(1-g^{-1}(\mathbf{x})\right) x^{n}+g^{-1}(\mathbf{x}) f(\mathbf{x}), \\
d(\mathbf{x})=g^{-1}(\mathbf{x}) d_{s} .
\end{gathered}
$$

Assumption 1 (see $[29,30])$. Assume that $f(\mathbf{x}), g(\mathbf{x})$, and $d_{s}$ satisfy $|f(\mathbf{x})| \leq \mu<\infty, 0<g_{\min } \leq g(\mathbf{x}) \leq g_{\max }<\infty$, and $|d| \leq \kappa$, respectively, for all $\mathbf{x} \in \mathbf{U}_{\mathbf{x}} \subset \mathfrak{R}^{n}$.

Where $\mu, g_{\min }, g_{\max }$, and $\kappa$ are known constants. The control problem is to force the system output $y$ to follow a given bounded reference signal $y_{d}$.

Define the tracking error as

$$
e=y_{d}-y
$$

\section{Takagi-Sugeno (T-S) Fuzzy Model}

Fuzzy logic systems address the imprecision of the input and output variables directly by defining them with fuzzy numbers (and fuzzy sets) that can be expressed in linguistic terms. The basic configuration of the Takagi and Sugeno $[5,8,31]$ system includes a rule base, which consists of a collection of fuzzy IF-THEN rules in the following form:

Plant Rule $r$ :

$$
\begin{aligned}
& \text { IF } x_{1} \text { is } B_{1}^{r} \text { and } \cdots \text { and } x_{n} \text { is } B_{n}^{r} \text {, } \\
& \text { THEN } y_{r}=a_{0}^{r}+a_{1}^{r} x_{1}+\cdots+a_{n}^{r} x_{n}=\theta_{r}^{T} \mathbf{x},
\end{aligned}
$$

where $B_{i}^{r}$ are fuzzy sets and $\theta_{r}^{T}=\left[a_{0}^{r}, a_{1}^{r}, \ldots, a_{n}^{r}\right]$ is a vector of the adjustable factors on the consequence part of the fuzzy rule, and the input vector $\mathbf{x}=\left[1, x_{1}, \ldots, x_{n}\right] \in R^{n}$. Let $i=1,2, \ldots, n$ denote the number of input for fuzzy logic system, and let $r=1,2, \ldots, m$ denote the number of the fuzzy IF-THEN rules. By using the singleton fuzzification, product inference and centre average defuzzication, the output value of the fuzzy system is

$$
y(\mathbf{x})=\frac{\sum_{r=1}^{m} y^{r}\left(\prod_{i=1}^{n} \mu_{B_{i}^{r}}\left(x_{i}\right)\right)}{\sum_{r=1}^{m}\left(\prod_{i=1}^{n} \mu_{B_{i}^{r}}\left(x_{i}\right)\right)},
$$

where $\mu_{B_{i}^{r}}\left(x_{i}\right)$ is the membership function value of the fuzzy variable $x_{i}$ and $\prod_{i=1}^{n} \mu_{B_{i}^{r}}\left(x_{i}\right)$ is the true value of the $r$ th implication. Equation (8) can be rewritten as

$$
y(\mathbf{x})=\theta^{T} \xi(\mathbf{x}),
$$

where $\theta^{T}=\left[\theta_{1}^{T}, \theta_{2}^{T}, \ldots, \theta_{m}\right]$ is an adjustable parameter vector, $\xi(\mathbf{x})^{T}=\left[\xi^{1}(\mathbf{x}), \ldots, \xi^{m}(\mathbf{x})\right]$ is a fuzzy basis function vector in which, $\xi^{r}(\mathbf{x}), r=1,2, \ldots, m$,

$$
\xi^{r}(\mathbf{x})=\frac{\prod_{i=1}^{n} \mu_{B_{i}^{r}}\left(x_{i}\right)}{\sum_{r=1}^{m} \prod_{i=1}^{n} \mu_{B_{i}^{r}}\left(x_{i}\right)} .
$$

The aforementioned fuzzy system has been shown to be capable of universally approximating well-defined functions over a compact set to arbitrary degree of accuracy. For smooth nonlinear functions $F(\mathbf{x}), d(\mathbf{x})$, they can be approximated by

$$
\begin{aligned}
& F(\mathbf{x})=\theta_{f}^{* T} \Psi(\mathbf{x})+\varepsilon, \\
& d(\mathbf{x})=\theta_{d}^{* T} \Xi(\mathbf{x})+\sigma,
\end{aligned}
$$

where $\varepsilon$ and $\sigma$ are the fuzzy approximations and $\theta_{f}^{*}$, and $\theta_{d}^{*}$ are optimal weight vectors.

And whose estimates are given by

$$
\begin{aligned}
& F\left(\frac{\mathbf{x}}{\widehat{\theta}_{f}}\right)=\widehat{\theta}_{f}^{T} \Psi(\mathbf{x}), \\
& d\left(\frac{\mathbf{x}}{\widehat{\theta}_{d}}\right)=\widehat{\theta}_{d}^{T} \Xi(\mathbf{x}) .
\end{aligned}
$$

\section{Adaptive Fuzzy Sliding Mode Controller Design}

In this section, a systematic methodology is presented for the design of stable adaptive fuzzy sliding mode controller, 
the control law and the weight adaptation rules are developed, guaranteeing the uniform ultimate boundedness of the tracking error with respect to an arbitrary small set around the origin. Additionally, the boundedness of all signals involved in the closed-loop configuration is ensured. The resetting scheme is introduced, performing on the weight estimates $\theta_{f}, \theta_{d}$ to guarantee the validity of the control law.

If we consider the system given by (4), the sliding surface can be defined by

$$
S=a_{n-1} e+\cdots+a_{1} e^{n-2}+e^{n-1} .
$$

The elements of the sliding surface are chosen such that the polynomial $a_{n-1} p^{n-1}+a_{n-2} p^{n-2}+\cdots+a_{0}$ is strictly Hurwitz [32] (here $p$ denotes the complex Laplace transform variable).

We propose to choose " $a$ " as follows [33]:

$$
a_{i}=\frac{M_{i}}{\left|e^{n-1-i}\right|+\eta_{i}} ; \quad i=1, \ldots, n-1,
$$

where $M_{i}$ is a given positive scalar, and $\eta_{i}$ is positive constant low value.

Note that $M / \eta$ represents the slope of sliding along the surface when it is reached by the system.

By using the tracking error defined by (6), the time derivative of (13) is

$$
\begin{gathered}
\dot{S}=\sum_{i=1}^{n-1} a_{n-i} e^{i}+\sum_{i=1}^{n-1} \dot{a}_{n-i} e^{i-1}+e^{n} \\
\dot{S}=y_{d}^{n}-y^{n}+\sum_{i=1}^{n-1} a_{n-i} e^{i}+\sum_{i=1}^{n-1} \dot{a}_{n-i} e^{i-1} \\
=y_{d}^{n}-F(\mathbf{x})-u-d(\mathbf{x})+\sum_{i=1}^{n-1} a_{n-i} e^{i}+\sum_{i=1}^{n-1} \dot{a}_{n-i} e^{i-1} \\
=y_{d}^{n}-F(\mathbf{x})-u-d(\mathbf{x})+\mathbf{A e},
\end{gathered}
$$

where $n$ is the $n$th derivative of the system, and $\mathbf{A}=$ $\left[\dot{a}_{n-1}, a_{n-1}, \dot{a}_{n-2}, \ldots, \dot{a}_{1}, a_{1}\right], \mathbf{e}=\left[e, \ldots, e^{n-3} ; e^{n-2}, e^{n-2} ; e^{n-1}\right]^{T}$.

Assumption 2. Let $\mathbf{x}$ belong to a compact set $\Omega_{x}$. The optimal weight vectors $\theta_{f}^{*}$ and $\theta_{d}^{*}$ are defined as

$$
\begin{gathered}
\theta_{f}^{*}=\underset{\widehat{\theta}_{f} \in \Omega_{f}}{\arg \min }\left[\sup _{x \in \Omega_{x}}\left[F\left(\frac{\mathbf{x}}{\widehat{\theta}_{f}}\right)-F(\mathbf{x})\right]\right], \\
\theta_{d}^{*}=\underset{\hat{\theta}_{d} \in \Omega_{d}}{\arg \min }\left[\sup _{x \in \Omega_{x}}\left[d\left(\frac{\mathbf{x}}{\hat{\theta}_{d}}\right)-d(\mathbf{x})\right]\right] .
\end{gathered}
$$

And define the constraint sets that the parameters concerned belong to

$$
\begin{aligned}
& \Omega_{f}=\left\{\theta_{f} \mid\left\|\theta_{f}\right\| \leq M_{f}\right\}, \\
& \Omega_{d}=\left\{\theta_{d} \mid\left\|\theta_{d}\right\| \leq M_{d}\right\},
\end{aligned}
$$

where $M_{f}$ and $M_{g}$ are design parameters.
We assume that $\widehat{\theta}_{f}, \widehat{\theta}_{d}$, and $x$ never reach the boundaries $\Omega_{f}, \Omega_{d}$, and $\Omega_{x}$. We can define the minimum approximation errors as

$$
\begin{aligned}
& \varepsilon=F(\mathbf{x})-F\left(\frac{\mathbf{x}}{\theta_{f}^{*}}\right), \\
& \sigma=d(\mathbf{x})-d\left(\frac{\mathbf{x}}{\theta_{d}^{*}}\right) .
\end{aligned}
$$

It is assumed that minimum approximation errors are bounded for all $x \in \Omega_{x}$ :

$$
|\varepsilon| \leq \bar{\varepsilon}, \quad|\sigma| \leq \bar{\sigma}, \quad \forall x \in \Omega_{x}
$$

The upper bound $\bar{\varepsilon}, \bar{\sigma}$ can be reduced arbitrarily. But this choice is not always easy, that is our aim in this work to estimate them by adaptive laws, which guarantee the stability of the closed loop system.

The role of the fuzzy systems $F\left(\mathbf{x} / \widehat{\theta}_{f}\right)$ and $d\left(\mathbf{x} / \widehat{\theta}_{d}\right)$ is to represent the unknown functions using the input-output measurement of the target system. Also, a corrective controller is defined to guarantee the stability of the closed-loop control system and compensate the approximation errors. A direct adaptive control law can be chosen as

$$
u=\left(\left|F\left(\frac{\mathbf{x}}{\widehat{\theta}_{f}}\right)\right|+\left|d\left(\frac{\mathbf{x}}{\widehat{\theta}_{d}}\right)\right|+y_{d}^{n}+\lambda+\mathbf{A e}+\hat{\bar{\varepsilon}}+\hat{\bar{\sigma}}\right) \operatorname{sgn}(S),
$$

where $\lambda$ is a strictly positive constant, and $\widehat{\bar{\varepsilon}}, \widehat{\bar{\sigma}}$ are estimates of $\bar{\varepsilon}, \bar{\sigma}$, and

$$
\operatorname{sgn}(S)= \begin{cases}1 & \text { if } S>0 \\ 0 & \text { if } S=0 \\ -1 & \text { if } S<0\end{cases}
$$

Theorem 1. Consider the nonlinear system described by (4), and suppose that Assumptions 1 and 2 are satisfied. The control law is provided by (20), and the parameters adaptation laws are given by

$$
\begin{gathered}
\dot{\hat{\theta}}_{f}=\gamma_{f} \Psi_{f}(\mathbf{x}) S, \\
\dot{\hat{\theta}}_{d}=\gamma_{d} \Xi_{d}(\mathbf{x}) S, \\
\dot{\overline{\bar{\varepsilon}}}=S \gamma_{\varepsilon}, \\
\dot{\overline{\bar{\sigma}}}=S \gamma_{\sigma} .
\end{gathered}
$$

Then, the desired tracking performance can be achieved as $S$ becomes asymptotically stable and all adaptation parameters remain bounded. 
Proof. Taking into account the minimum approximation errors (18) and control law (20), the sliding surface (15) can be rewritten as

$$
\begin{aligned}
\dot{S}=y_{d}^{n}-\left(\left|F\left(\frac{\mathbf{x}}{\widehat{\theta}_{f}}\right)\right|+\left|d\left(\frac{\mathbf{x}}{\widehat{\theta}_{d}}\right)\right|\right. & \\
& \left.+y_{d}^{n}+l+\mathbf{A e}+\hat{\bar{e}}+\hat{\bar{s}}\right) \operatorname{sgn}(S) \\
& -F(\mathbf{x})-d(\mathbf{x})+\mathbf{A e} .
\end{aligned}
$$

Defining the parameters errors $\widetilde{\theta}_{f}=\widehat{\theta}_{f}-\theta_{f}^{*}, \widetilde{\theta}_{d}=\widehat{\theta}_{d}-\theta_{d}^{*}$.

We choose the Lyapunov function candidate as follows:

$$
\begin{gathered}
V=\frac{1}{2} S^{2}+\frac{1}{2 \gamma_{f}} \widehat{\theta}_{f}^{T} \widehat{\theta}_{f}+\frac{1}{2 \gamma_{d}} \widehat{\theta}_{d}^{T} \widehat{\theta}_{d}+\frac{1}{2 \gamma_{e}} \hat{\bar{e}}^{2}+\frac{1}{2 \gamma_{s}} \widehat{\overline{\mathcal{s}}}^{2} \\
\tilde{\bar{\varepsilon}}=\bar{\varepsilon}-\hat{\bar{\varepsilon}}, \quad \tilde{\bar{\sigma}}=\bar{\sigma}-\hat{\bar{\sigma}},
\end{gathered}
$$

where $\gamma_{f}, \gamma_{d}, \gamma_{\varepsilon}$, and $\gamma_{\sigma}$ are positive constants. The time derivative of (24) can be obtained as follows:

$$
\begin{aligned}
& \dot{V}=S \dot{S}+\frac{1}{\gamma_{f}} \widetilde{\theta}_{f}^{T} \dot{\widetilde{\theta}}_{f}+\frac{1}{\gamma_{d}} \widetilde{\theta}_{d}^{T} \dot{\tilde{\theta}}_{d}+\frac{1}{\gamma_{\varepsilon}} \tilde{\bar{\varepsilon}} \dot{\bar{\varepsilon}}+\frac{1}{\gamma_{\sigma}} \tilde{\bar{\sigma}} \dot{\bar{\sigma}} \\
& =S\left(y_{d}^{n}-F(\mathbf{x})-\left(\left|F\left(\frac{\mathbf{x}}{\widehat{\hat{\theta}}_{f}}\right)\right|+\left|d\left(\frac{\mathbf{x}}{\widehat{\hat{\theta}}_{d}}\right)\right|+y_{d}^{n}+\lambda+\mathbf{A e}\right.\right. \\
& +\hat{\bar{\varepsilon}}+\hat{\bar{\sigma}}) \operatorname{sgn}(S)-d(\mathbf{x})+\mathbf{A e}) \\
& +\frac{1}{\gamma_{f}} \widetilde{\theta}_{f}^{T} \dot{\tilde{\theta}}_{f}+\frac{1}{\gamma_{d}} \widetilde{\theta}_{d}^{T} \dot{\tilde{\theta}}_{d}+\frac{1}{\gamma_{\varepsilon}} \tilde{\bar{\varepsilon}} \dot{\bar{\varepsilon}}+\frac{1}{\gamma_{\sigma}} \tilde{\bar{\sigma}} \dot{\bar{\sigma}} \\
& \leq|S||\mathbf{A e}|+|S| y_{d}^{n}-S\left(\left|F\left(\frac{\mathbf{x}}{\hat{\theta}_{f}}\right)\right|+\left|d\left(\frac{\mathbf{x}}{\widehat{\theta}_{d}}\right)\right|+\ddot{y}_{d}\right. \\
& +\lambda+\mathbf{A e}+\hat{\bar{\varepsilon}}+\hat{\bar{\sigma}}) \operatorname{sgn}(S) \\
& -S F(\mathbf{x})-S d(\mathbf{x})+\frac{1}{\gamma_{f}} \widetilde{\theta}_{f}^{T} \dot{\tilde{\theta}}_{f}+\frac{1}{\gamma_{d}} \widetilde{\theta}_{d}^{T} \dot{\tilde{\theta}}_{d}+\frac{1}{\gamma_{\varepsilon}} \widetilde{\bar{\varepsilon}} \dot{\bar{\varepsilon}}+\frac{1}{\gamma_{\sigma}} \tilde{\bar{\sigma}} \dot{\bar{\sigma}} \\
& =-\lambda|S|-|S|\left|F\left(\frac{\mathbf{x}}{\widehat{\hat{\theta}}_{f}}\right)\right|-|S|\left|d\left(\frac{\mathbf{x}}{\hat{\theta}_{d}}\right)\right|-|S| \hat{\bar{\varepsilon}} \\
& -|S| \widehat{\bar{\sigma}}-S F(\mathbf{x})-S d(\mathbf{x})
\end{aligned}
$$

$$
\begin{aligned}
& +\frac{1}{\gamma_{f}} \widetilde{\theta}_{f}^{T} \dot{\tilde{\theta}}_{f}+\frac{1}{\gamma_{d}} \widetilde{\theta}_{d}^{T} \dot{\tilde{\theta}}_{d}+\frac{1}{\gamma_{\varepsilon}} \widetilde{\bar{\varepsilon}} \dot{\bar{\varepsilon}}+\frac{1}{\gamma_{\sigma}} \widetilde{\bar{\sigma}} \dot{\bar{\sigma}} \\
& =-\lambda|S|-|S|\left|F\left(\frac{\mathbf{x}}{\widehat{\hat{\theta}}_{f}}\right)\right|-|S|\left|d\left(\frac{\mathbf{x}}{\widehat{\hat{\theta}}_{d}}\right)\right|-|S| \widehat{\bar{\varepsilon}} \\
& -|S| \hat{\bar{\sigma}}-S\left(F\left(\frac{\mathbf{x}}{\theta_{f}^{*}}\right)+\varepsilon\right) \\
& -S\left(d\left(\frac{\mathbf{x}}{\theta_{d}^{*}}\right)+\sigma\right)+\frac{1}{\gamma_{f}} \widetilde{\theta}_{f}^{T} \dot{\tilde{\theta}}_{f}+\frac{1}{\gamma_{d}} \widetilde{\theta}_{d}^{T} \dot{\tilde{\theta}}_{d} \\
& +\frac{1}{\gamma_{\varepsilon}} \widetilde{\bar{\varepsilon}} \dot{\bar{\varepsilon}}+\frac{1}{\gamma_{\sigma}} \tilde{\bar{\sigma}} \dot{\bar{\sigma}} \\
& \leq-\lambda|S|-|S|\left|F\left(\frac{\mathbf{x}}{\widehat{\hat{\theta}}_{f}}\right)\right|-|S|\left|d\left(\frac{\mathbf{x}}{\widehat{\widehat{\theta}}_{d}}\right)\right|-|S| \hat{\bar{\varepsilon}}-|S| \hat{\bar{\sigma}} \\
& +|S|\left|F\left(\frac{\mathbf{x}}{\widehat{\theta}_{f}}\right)\right|-|S|\left|d\left(\frac{\mathbf{x}}{\widehat{\theta}_{d}}\right)\right| \\
& -S \tilde{\theta}_{f} \Psi(\mathbf{x})-S \tilde{\theta}_{d} \Xi(\mathbf{x})+|S| \bar{\varepsilon}+|S| \bar{\sigma}+\frac{1}{\gamma_{f}} \tilde{\theta}_{f}^{T} \dot{\tilde{\theta}}_{f} \\
& +\frac{1}{\gamma_{d}} \tilde{\theta}_{d}^{T} \dot{\tilde{\theta}}_{d}+\frac{1}{\gamma_{\varepsilon}} \widetilde{\bar{\varepsilon}} \dot{\bar{\varepsilon}}+\frac{1}{\gamma_{\sigma}} \widetilde{\bar{\sigma}} \dot{\bar{\sigma}} \\
& =-\lambda|S|-S \widetilde{\theta}_{f} \Psi(\mathbf{x})-S \widetilde{\theta}_{d} \Xi(\mathbf{x})+|S| \tilde{\bar{\varepsilon}}+|S| \tilde{\bar{\sigma}} \\
& +\frac{1}{\gamma_{f}} \widetilde{\theta}_{f}^{T} \dot{\tilde{\theta}}_{f}+\frac{1}{\gamma_{d}} \widetilde{\theta}_{d}^{T} \dot{\tilde{\theta}}_{d}+\frac{1}{\gamma_{\varepsilon}} \widetilde{\bar{\varepsilon}} \dot{\bar{\varepsilon}}+\frac{1}{\gamma_{\sigma}} \tilde{\bar{\sigma}} \dot{\bar{\sigma}} \\
& =-\lambda|S|+\widetilde{\theta}_{f}^{T}\left(-S \Psi_{f}(\mathbf{x})+\frac{1}{\gamma_{f}} \dot{\tilde{\theta}}_{f}\right) \\
& +\tilde{\theta}_{d}^{T}\left(-S \Xi_{d}(\mathbf{x}) u+\frac{1}{\gamma_{d}} \dot{\tilde{\theta}}_{d}\right) \\
& +\tilde{\bar{\varepsilon}}\left(S+\frac{1}{\gamma_{\varepsilon}} \dot{\bar{\varepsilon}}\right)+\tilde{\bar{\sigma}}\left(S+\frac{1}{\gamma_{d}} \dot{\overline{\bar{\sigma}}}\right) .
\end{aligned}
$$

Choosing a fuzzy rule adaptive method as

$$
\begin{gathered}
\dot{\tilde{\theta}}_{f}=\gamma_{f} \Psi_{f}(\mathbf{x}) S, \\
\dot{\tilde{\theta}}_{g}=\gamma_{d} \Xi_{d}(\mathbf{x}) S, \\
\dot{\overline{\tilde{\varepsilon}}}=-S \gamma_{\varepsilon}, \\
\dot{\bar{\sigma}}=-S \gamma_{\sigma}
\end{gathered}
$$


or equivalently, by definition

$$
\begin{gathered}
\dot{\widehat{\theta}}_{f}=\gamma_{f} \Psi_{f}(\mathbf{x}) S, \\
\dot{\widehat{\theta}}_{g}=\gamma_{d} \Xi_{d}(\mathbf{x}) S, \\
\dot{\overline{\bar{\varepsilon}}}=S \gamma_{\varepsilon}, \\
\dot{\overline{\bar{\sigma}}}=S \gamma_{\sigma}
\end{gathered}
$$

yields

$$
\dot{V}=-\lambda|S|
$$

Integrating both sides of (28), we have $\int_{0}^{\infty} \dot{V} d t \leq$ - $\int_{0}^{\infty}(1 / 2)|S| d t$, and thus, the following equation holds:

$$
\int_{0}^{\infty}|S| d t \leq 2(V(0)-V(\infty)) .
$$

As $V(0)$ is bounded and also $0 \leq V(\infty) \leq V(0)$ from (28), $\int_{0}^{\infty}|S| d t$ is also bounded from (29). Using Barbalat's lemma, $[19,34]|S| \rightarrow 0$ for $t \rightarrow \infty$.

From the moment where the sliding surface is designed and constructed to be attractive, we can also see that $\lim _{t \rightarrow \infty} e=0$. Therefore, the closed-loop system is asymptotically stable and the position tracking objective is achieved. The modified projection adaptive laws are given in [7].

\section{Simulation Example}

We illustrate the validity of the design approach by an example of robot arm tracking control with a single degree of freedom as Figure 1 shows.

The dynamic equations of such a system are given by

$$
\begin{gathered}
x^{(3)}=f(\mathbf{x})+g(\mathbf{x}) u+d, \\
y=x,
\end{gathered}
$$

where

$$
\begin{gathered}
f(\mathbf{x})=-\frac{r}{L} x_{3}-\left(\frac{g}{l} \cos \left(x_{1}\right)+\frac{K_{b} N^{2} K_{t}}{L m l^{2}}\right) x_{2}-\frac{r g}{L l} \sin \left(x_{1}\right), \\
g(\mathbf{x})=\frac{K_{t} N}{L m l^{2}}, \\
\mathbf{x}=\left[x_{1}, x_{2}, x_{3}\right]^{T},
\end{gathered}
$$

where $x_{1}$ is the angular position ( $\left.\mathrm{rad}\right), x_{2}$ is the angular velocity $(\mathrm{rad} / \mathrm{s}), x_{3}$ is the angular acceleration $\left(\mathrm{rad} / \mathrm{s}^{2}\right), u(t)$ is the applied force (control signal) $(\mathrm{N})$, and $d$ is the external disturbance. The simulation parameters are given in Table 1.

According to (30), we choose the sliding surface as $S=$ $e^{(2)}+a_{2} e^{(1)}+a_{1} e$. The following parameters are chosen so that

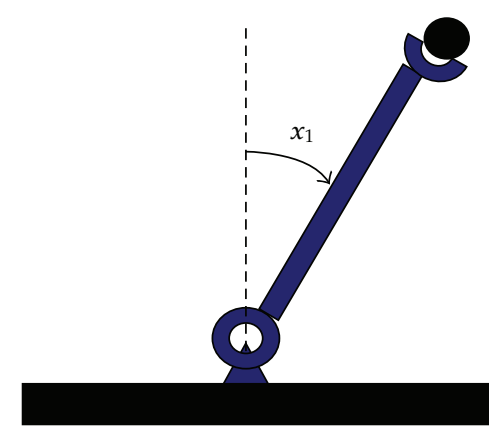

Figure 1: Robot arm.

TABLE 1: Simulation parameters.

\begin{tabular}{lc}
\hline Mass of the pole & $m=5 \mathrm{~kg}$ \\
The half-length of the pole & $l=0.5 \mathrm{~m}$ \\
The acceleration due to gravity & $G=9.8$ \\
Resistance & $r=1.5$ \\
Inductance & $L=0.5$ \\
Electromotive force constant & $K_{b}=0.2$ \\
Constant torque motor & $K_{t}=0.3$ \\
Reduction ratio & $N=60$ \\
\hline
\end{tabular}

the characteristic function of the surface is the negative real part

$$
M_{1}=8, \quad \eta_{1}=1, \quad M_{2}=15 \quad \eta_{2}=1 .
$$

To construct two fuzzy logic systems, $F\left(\mathbf{x} / \widehat{\theta}_{f}\right)$ and $d\left(\mathbf{x} / \widehat{\theta}_{d}\right)$ as given in (12), the initial consequent parameters of fuzzy rules are chosen randomly in the interval $[-1.2,1.2]$. The initial values of $\mathbf{x}$ are given as $\left[\begin{array}{lll}0 & 0 & 0\end{array}\right]^{T}$.

This interval will be sufficiently covered by three membership functions for position, velocity, and angular acceleration. Then, we have 27 rules.

Let the learning rate $\gamma_{f}=0.05, \gamma_{d}=20, \gamma_{\varepsilon}=100, \gamma_{\sigma}=$ $500, k_{d}=6$, and $M=40$.

The control objective is to maintain the system to track the desired angle trajectory, $y_{d}=\sin (t)$, and to test the proposed control, we introduced parametric variations and external disturbances given by $\Delta m=0.1 \sin (x), d=0.125 \times \sin (2 t)$, respectively.

Figures 2-5 show the simulation results obtained in the case where the system is subjected to external disturbances and parametric variations. Figures 2, 3, and 4 show the rapid convergence of the system output to the reference signal. In Figure 5, we can see that the control signal is smooth and that the actual and desired trajectories are superposed, after a short transitional arrangement whereby the error is significant between the two outputs, this is due to disturbances, initial conditions, and initialization of adjustable parameters.

Figures 2-4 show that the effect of parametric perturbations is negligible, with less stress to the control level despite greater external disturbances. Similarly, the results obtained in [35] show that the tracking error is about $8 \%$ whereas it is less than $2,5 \%$ in our case. 


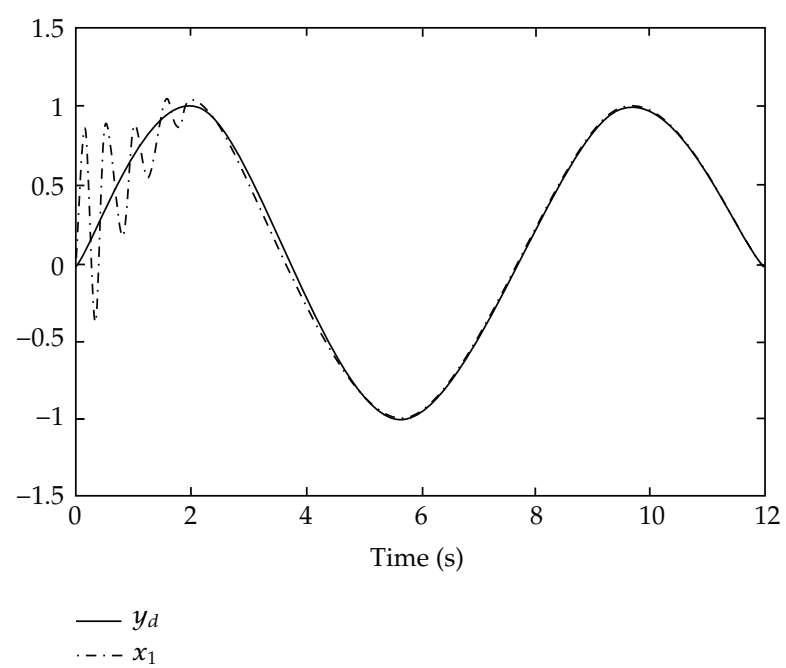

FIGURE 2: Trajectories of the state $x_{1}(t)$ of tracking control of the desired $y_{d}(t)$ for the robot arms.

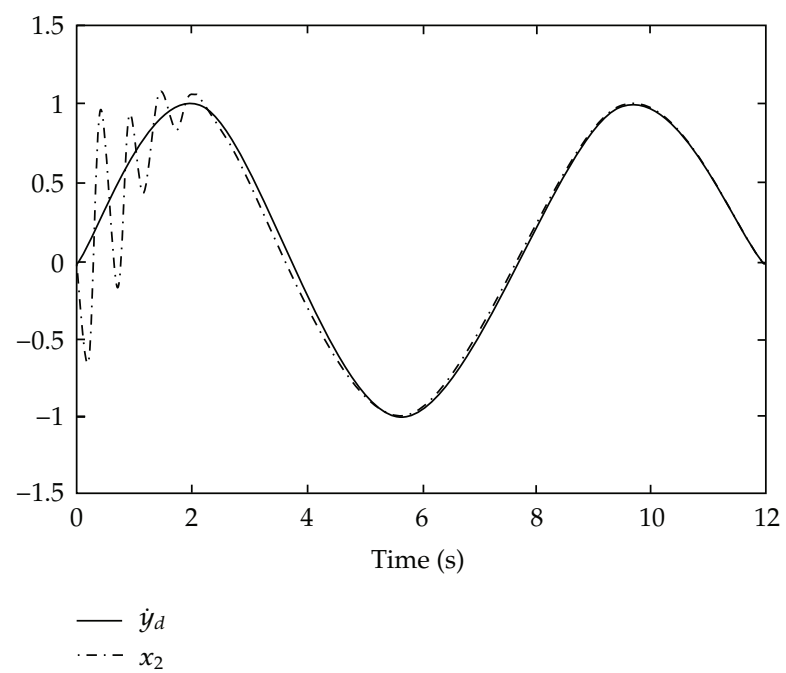

FIgURE 3: Trajectories of the state $x_{2}(t)$ of tracking control of the desired $\dot{y}_{d}(t)$ for the robot arm.

It can be seen in Figures 2-5 that the advantage of our controller is its ability to eliminate the effect of fluctuations in the transient response with less effort on the control law; moreover, an estimation of the upper bound of error is performed without needing their prior knowledge, which allows the control law to be less restrictive regarding the conditions of stability.

\section{Conclusion}

In this paper, the output tracking control problem has been considered for a class of uncertain nonlinear systems. The unknown functions in systems are not linearly parameterized and have no a priori knowledge of the bounded functions. Fuzzy logic systems are used to approximate these unknown nonlinear functions. By sliding mode design technique, the

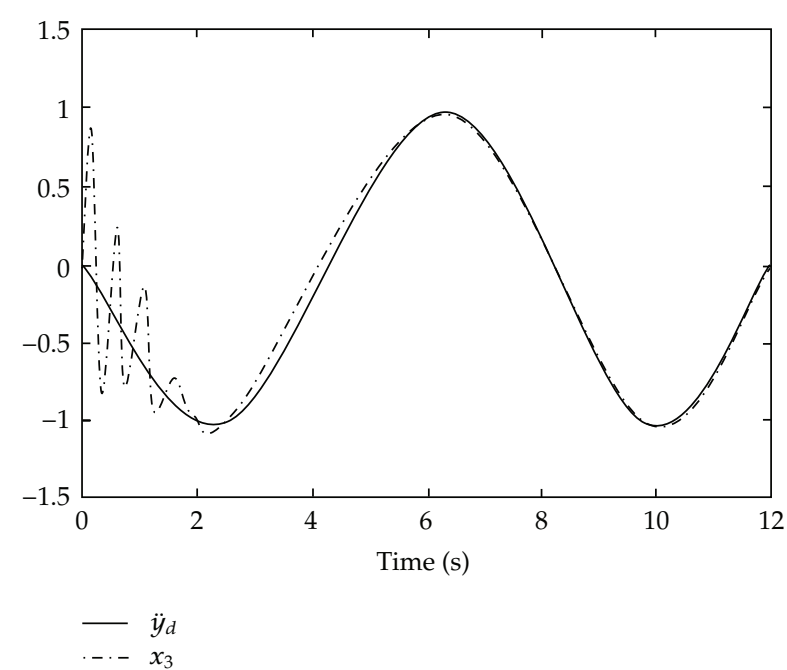

FIGURE 4: Trajectories of the state $x_{3}(t)$ of tracking control of the desired $\ddot{y}_{d}(t)$ for the robot arm.

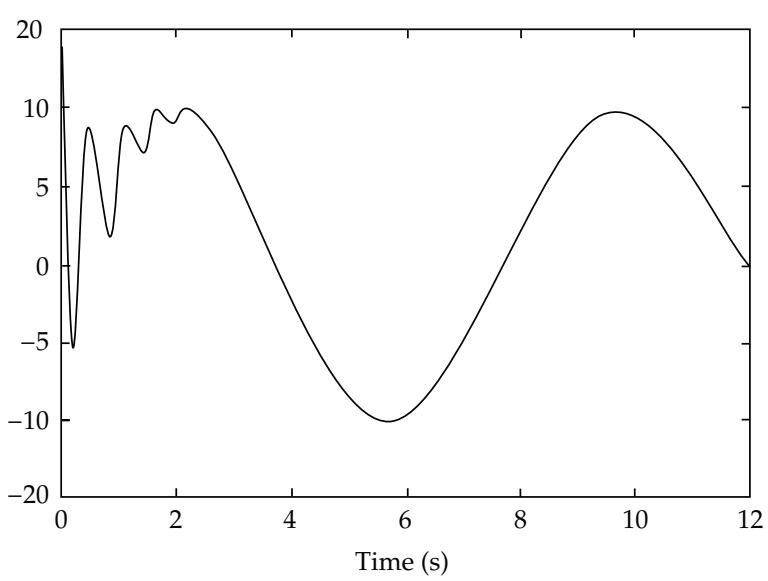

FIgURE 5: Trajectories of the control input $u(t)$ of the tracking control for robot arm.

adaptive fuzzy tracking control scheme has been developed for nonlinear systems. The proposed controllers guarantee that the outputs of the closed-loop system follow the reference signals, and achieve uniform ultimate boundedness of all the signals in the closed-loop system. It is proved in theory and shown in simulation that the closed-loop system is stable and the output tracks the given reference signal satisfactorily. Future work will deal with the delay systems in the type 2 fuzzy systems taking into account uncertainties and a novel nonlinearity sliding mode surface and an application to a real process.

\section{References}

[1] S. Bououden, S. Filali, and K. Kemih, "Adaptive fuzzy tracking control for unknown nonlinear systems," International Journal of Innovative Computing, Information and Control, vol. 6, no. 2, pp. 541-549, 2010. 
[2] M. Chadli and A. El Hajjaji, "observer-based robust fuzzy control of nonlinear systems with parametric uncertainties," Fuzzy Sets and Systems, vol. 157, no. 9, pp. 1276-1281, 2006.

[3] M. Chadli, "On the stability analysis of uncertain fuzzy models," International Journal of Fuzzy Systems, vol. 8, no. 4, pp. 224-231, 2006.

[4] Y. J. Liu, W. Wang, S. C. Tong, and Y. S. Liu, "Robust adaptive tracking control for nonlinear systems based on bounds of fuzzy approximation parameters," IEEE Transactions on Systems, Man, and Cybernetics A, vol. 40, no. 1, pp. 170-184, 2010.

[5] K. Tanaka, H. Ohtake, and H. O. Wang, "A descriptor system approach to fuzzy control system design via fuzzy Lyapunov functions," IEEE Transactions on Fuzzy Systems, vol. 15, no. 3, pp. 333-341, 2007.

[6] L. Wu, X. Su, P. Shi, and J. Qiu, "Model approximation for discrete-time state-delay systems in the TS fuzzy framework," IEEE Transactions on Fuzzy Systems, vol. 19, no. 2, pp. 366-378, 2011.

[7] Y. C. Chang, "Robust tracking control for nonlinear MIMO systems via fuzzy approaches," Automatica, vol. 36, no. 10, pp. 1535-1545, 2000.

[8] Y. H. Chien, W. Y. Wang, Y. G. Leu, and T. T. Lee, "Robust adaptive controller design for a class of uncertain nonlinear systems using online T-S fuzzy-neural modeling approach," IEEE Transactions on Systems, Man, and Cybernetics B, vol. 41, no. 2, pp. 542-552, 2011.

[9] M. Krstic, I. Kanellakopoulos, and P. Koktovic, Nolinear and Adaptive Control, Wiley, New York, NY, USA, 1995.

[10] X. Zhang, C. Wang, D. Li, X. Zhou, and D. Yang, "Robust stability of impulsive Takagi-Sugeno fuzzy systems with parametric uncertainties," Information Sciences, vol. 181, no. 23, pp. 52785290, 2011.

[11] R. Yang, Z. Zhang, and P. Shi, "Exponential stability on stochastic neural networks with discrete interval and distributed delays," IEEE Transactions on Neural Networks, vol. 21, no. 1, pp. 169-175, 2010.

[12] L. Wu, X. Su, P. Shi, and J. Qiu, "A new approach to stability analysis and stabilization of discrete-time T-S fuzzy timevarying delay systems," IEEE Transactions on Systems, Man, and Cybernetics B, vol. 41, no. 1, pp. 273-286, 2011.

[13] S. Islam and X. P. Liu, "Robust sliding mode control for robot manipulators," IEEE Transactions on Industrial Electronics, vol. 58, no. 6, pp. 2444-2453, 2011.

[14] C. C. Kung and T. H. Chen, "Observer-based indirect adaptive fuzzy sliding mode control with state variable filters for unknown nonlinear dynamical systems," Fuzzy Sets and Systems, vol. 155, no. 2, pp. 292-308, 2005.

[15] T. C. Kuo, Y. J. Huang, and S. H. Chang, "Sliding mode control with self-tuning law for uncertain nonlinear systems," ISA Transactions, vol. 47, no. 2, pp. 171-178, 2008.

[16] T. R. Oliveira, A. J. Peixoto, and L. Hsu, "Sliding mode control of uncertain multivariable nonlinear systems with unknown control direction via switching and monitoring function," IEEE Transactions on Automatic Control, vol. 55, no. 4, pp. 1028-1034, 2010.

[17] V. I. Utkin, Sliding Modes and Their Application in Variable Structure Systems, MIR Publishers, Moscow, Russia, 1978.

[18] W. Y. Wang, M. L. Chan, C. C. J. Hsu, and T. T. Lee, "Ho tracking-based sliding mode control for uncertain nonlinear systems via an adaptive fuzzy-neural approach," IEEE Transactions on Systems, Man, and Cybernetics B, vol. 32, no. 4, pp. 483492, 2002.
[19] J. Fei and C. Batur, "A novel adaptive sliding mode control with application to MEMS gyroscope," ISA Transactions, vol. 48, no. 1, pp. 73-78, 2009.

[20] Y. J. Huang, T. C. Kuo, and S. H. Chang, "Adaptive sliding-mode control for nonlinear systems with uncertain parameters," IEEE Transactions on Systems, Man, and Cybernetics B, vol. 38, no. 2, pp. 534-539, 2008.

[21] V. Nekoukar and A. Erfanian, "Adaptive fuzzy terminal sliding mode control for a class of MIMO uncertain nonlinear systems," Fuzzy Sets and Systems, vol. 179, no. 1, pp. 34-49, 2011.

[22] Z. Xi, G. Feng, and T. Hesketh, "Piecewise integral slidingmode control for TS fuzzy systems," IEEE Transactions on Fuzzy Systems, vol. 19, no. 1, pp. 65-74, 2011.

[23] M. Yue, W. Sun, and P. Hu, "Sliding mode robust control for two-wheeled mobile robot with lower center of gravity," International Journal of Innovative Computing, Information and Control, vol. 7, no. 2, pp. 637-646, 2011.

[24] T. C. Lin and M. C. Chen, "Adaptive hybrid type-2 intelligent sliding mode control for uncertain nonlinear multivariable dynamical systems," Fuzzy Sets and Systems, vol. 171, no. 1, pp. 44-71, 2011.

[25] J. Zhang, P. Shi, and Y. Xia, "Robust adaptive sliding-mode control for fuzzy systems with mismatched uncertainties," IEEE Transactions on Fuzzy Systems, vol. 18, no. 4, pp. 700-711, 2010.

[26] Z. He, J. Wu, G. Sun, and C. Gao, "State estimation and sliding mode control of uncertain switched hybrid systems," International Journal of Innovative Computing Information and Control, vol. 8, no. 10, pp. 7143-7156, 2012.

[27] T. C. Lin, S. W. Chang, and C. H. Hsu, "Robust adaptive fuzzy sliding mode control for a class of uncertain discretetime nonlinear systems," International Journal of Innovative Computing Information and Control, vol. 8, no. 1, pp. 347-359, 2012.

[28] M. A. Demetriou and I. G. Rosen, "Variable structure model reference adaptive control of parabolic distributed parameter systems," in Proceedings of the American Control Conference, pp. 4371-4376, May 2002.

[29] L. X. Wang, A Course in Fuzzy Systems and Control, PrenticeHall, Englewood Cliffs, NJ, USA, 1997.

[30] C. H. Wang, H. L. Liu, and T. C. Lin, "Direct adaptive fuzzyneural control with state observer and supervisory controller for unknown nonlinear dynamical systems," IEEE Transactions on Fuzzy Systems, vol. 10, no. 1, pp. 39-49, 2002.

[31] A. Sala, T. M. Guerra, and R. Babuška, "Perspectives of fuzzy systems and control," Fuzzy Sets and Systems, vol. 156, no. 3, pp. 432-444, 2005.

[32] J. Wang and J. Hu, "Robust adaptive neural control for a class of uncertain non-linear time-delay systems with unknown deadzone non-linearity," IET Control Theory \& Applications, vol. 5, no. 15, pp. 1782-1795, 2011.

[33] Z. L. Liu, "Reinforcement adaptive fuzzy control of wing rock phenomena," IEE Proceedings: Control Theory and Applications, vol. 152, no. 6, pp. 615-620, 2005.

[34] J. E. Slotine and W. Li, Applied Nonlinear Control, Prentice-Hall, Upper Saddle River, NJ, USA, 1991.

[35] F. J. Lin, P. H. Shieh, and Y. C. Hung, "An intelligent control for linear ultrasonic motor using interval type-2 fuzzy neural network," IET Electric Power Applications, vol. 2, no. 1, pp. 3241, 2008. 


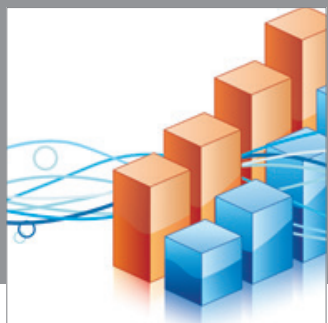

Advances in

Operations Research

mansans

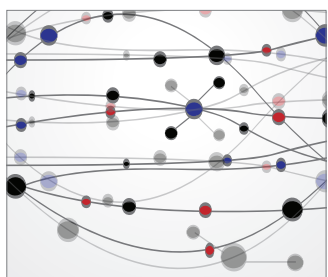

The Scientific World Journal
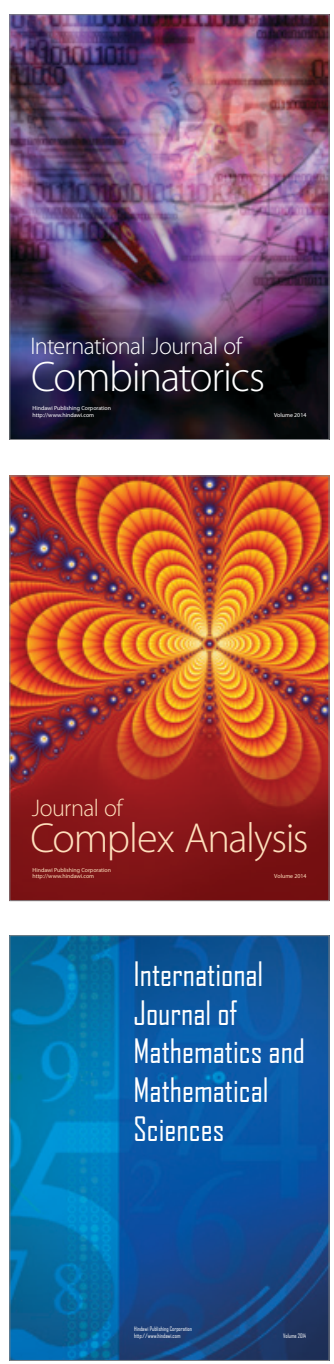
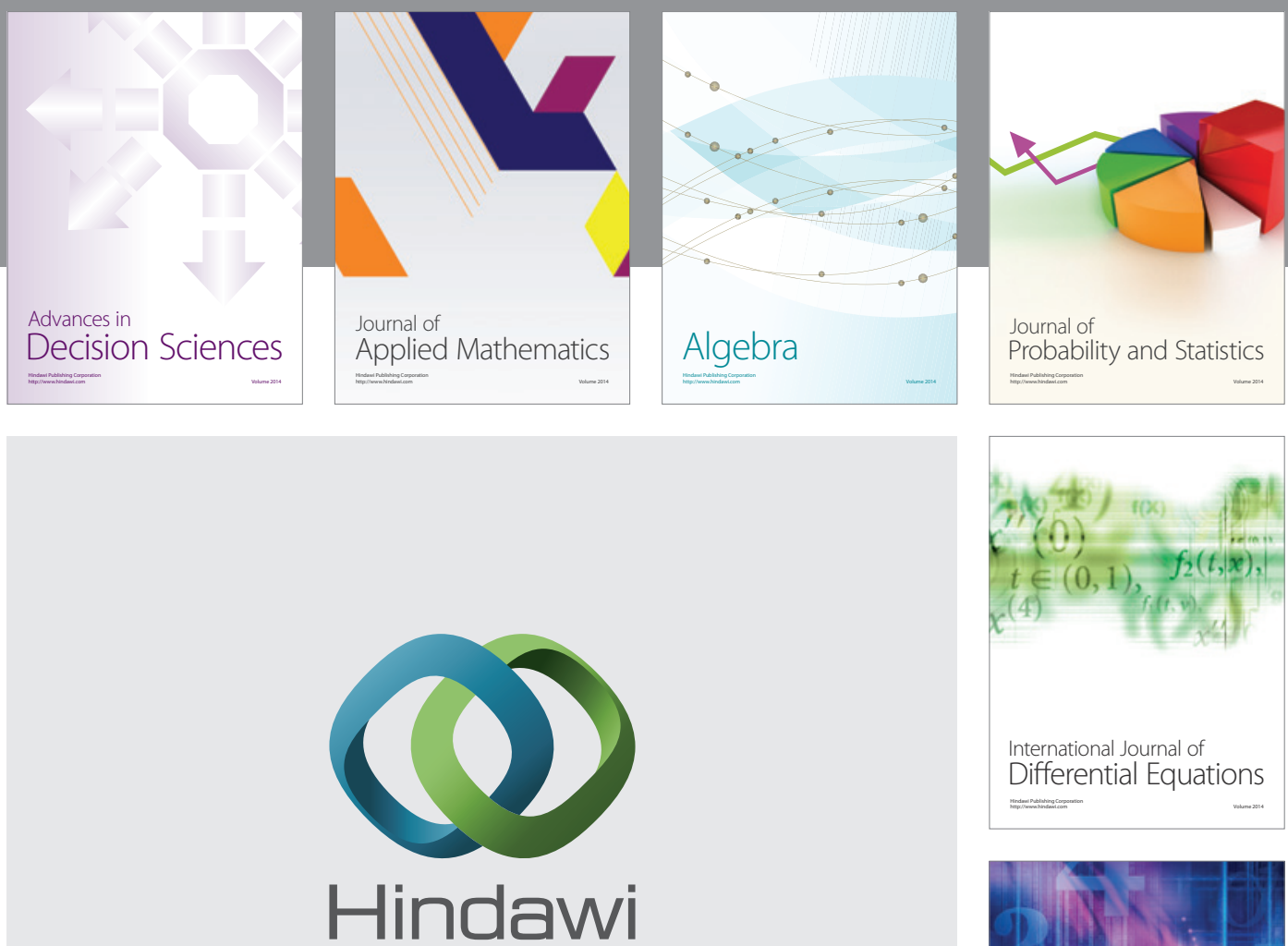

Submit your manuscripts at http://www.hindawi.com
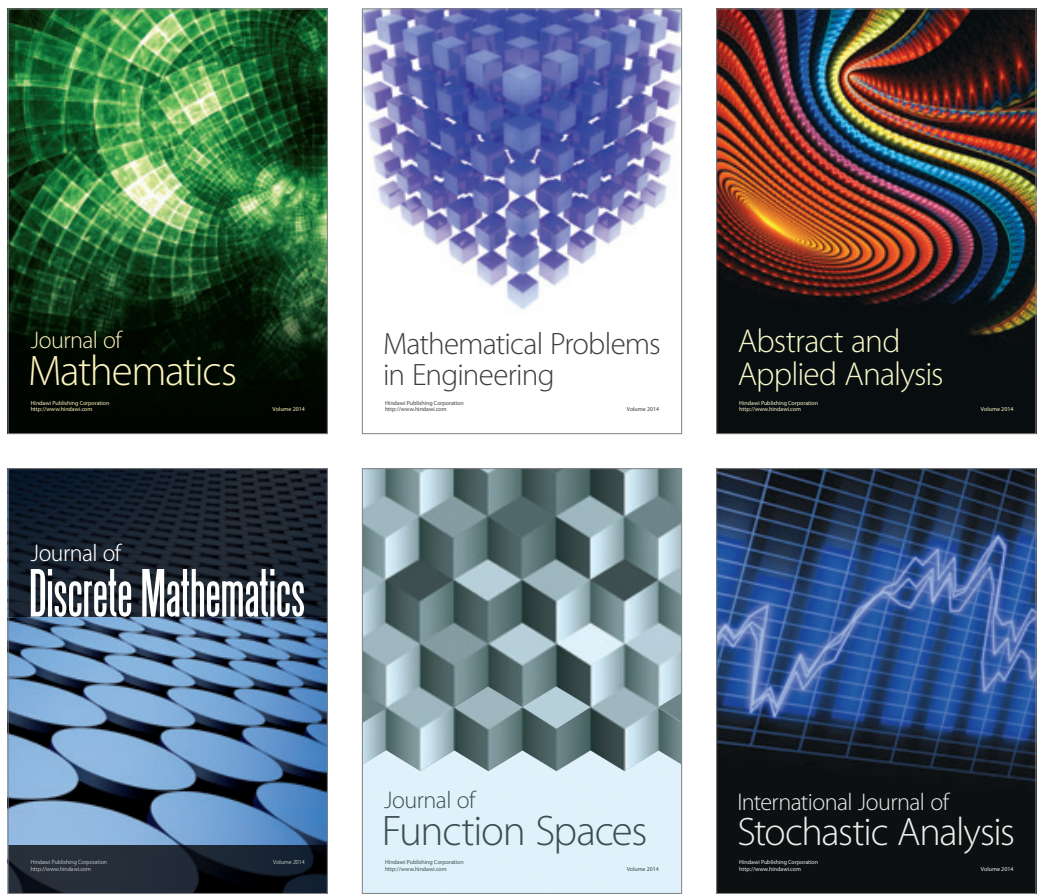

Journal of

Function Spaces

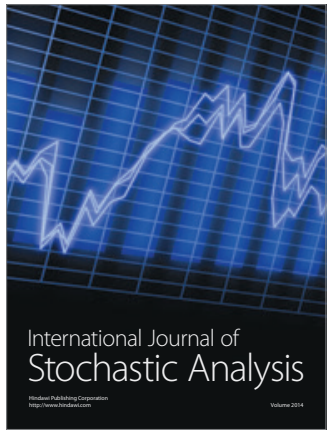

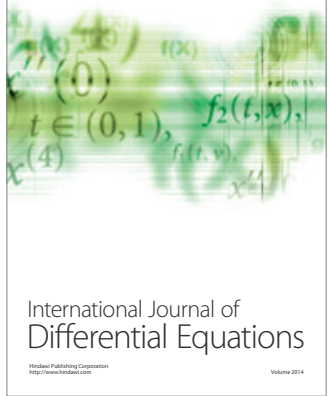
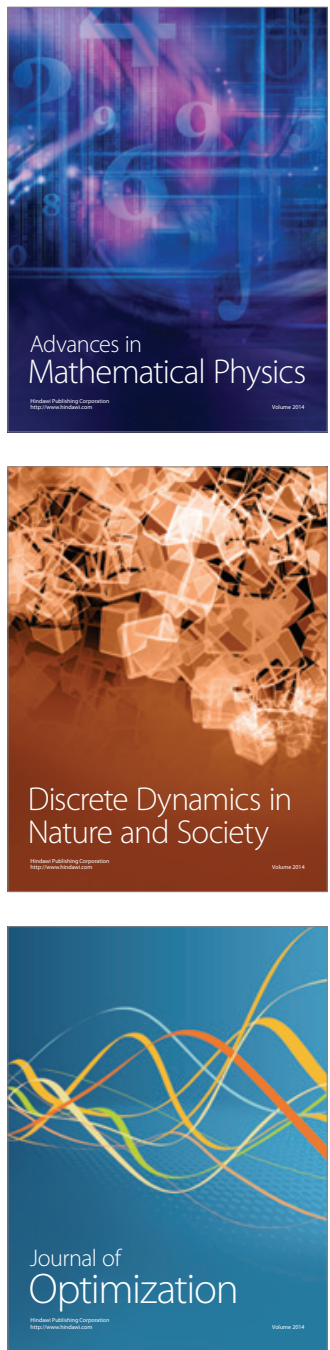